\title{
Spontaneous Pneumomediastinum- Review of 20 Cases
}

\author{
Mohammad Vaziri* \\ Thoracic Surgeon, Iran University of Medical Sciences, Iran \\ *Corresponding author: Mohammad Vaziri, Thoracic Surgeon, Iran University of Medical Sciences, Iran
}

\section{ARTICLE INFO}

Received: 幽 July 23, 2019

Published: August 01, 2019

Citation: Mohammad Vaziri. Spontaneous Pneumomediastinum- Review of 20 Cases. Biomed J Sci \& Tech Res 20(2)2019. BJSTR. MS.ID.003419.

\section{ABSTRACT}

Objective: Spontaneous pneumomediastinum, is defined as the presence of free air within the mediastinal cavity without an overt etiology. The purpose of this study is to review and discuss our experience with this condition.

Methods: A descriptive, retrospective study of 20 cases -14 men (70\%) and 6 women (30\%) -treated at our hospital for spontaneous pneumomediastinum from March 2001 to April 2018.

Results: The patients had a median age of 25 years (age range, 18 to 55 years).The most common presenting complaint was chest pain, occurring in 17 patients (85\%). Dyspnea was the next most common complaint (13 patients-65\%).The patients reported precipitating events in only half of the presentations which included coughing, sport activity and mild trauma. The initial diagnosis was almost correctly made in four patients $(20 \%)$ and in the remaining patients, the diagnosis was thought to be esophageal rupture, cardiac events and pneumothorax. All patients survived and were discharged from the hospital after a median time of 2 days (range, up to 4 days).

Conclusion: Spontaneous pneumomediastinum (SPM) has been described predominantly in young adult men, and its incidence appears to be increasing in more recent reports, but this may reflect greater physician awareness. The most important role of thoracic surgeon is to exclude the absence of more serious abnormalities, especially those who have a history of vomiting or retching.

\section{Introduction}

Spontaneous or Primary Pneumomediastinum which is a diagnosis of exclusion, was first described by Hamman in 1937. The pathophysiology of primary pneumomediastinum was first explained by Macklin to comprise of three essential steps:

a) A rapid increase in intrathoracic pressure results in alveolar rupture;

b) Small airway rupture leads to dissection of interstitial air along the tracheobronchial tree and perivascular planes; and

c) Once airway integrity has been compromised, a pressure gradient exists between the lung periphery and the mediastinum allowing air to dissect relatively freely into the central mediastinum $[1,2]$.

Primary pneumomediastinum has an estimated incidence of $1: 25,000$ to $1: 30,000$ and most frequently occurs in young healthy male patients who identify themselves as smokers [3,4]. Given its low incidence, it is unsurprising to find that the only published reports of this entity are of small case series. The objective of this study was to discuss our experience in the diagnosis and management of this clinical condition.

\section{Materials and Methods}

Between March 2001 to April 2018, all patients who were admitted to the Rasool Akram Hospital in Tehran with a diagnosis of spontaneous pneumomediastinum were reviewed. Their files were assessed retrospectively, and their demographic details, precipitating factors, comorbidities, symptoms, signs, treatments, and outcomes were reviewed. Exclusion criteria included evidence of a clear trigger for the pneumomediastinum, such as perforation of the tracheobronchial tree or the esophagus; iatrogenic factors (manipulation of the throat or larynx); pneumomediastinum occurring after thoracic or cardiac surgery; chest wounds and injuries; infection by gas-producing germs; or any disease involving the neck or abdomen 


\section{Results}

Twenty patients were identified from a review of the hospital records. There were 14 men (70\%) and 6 (30\%) women, and the patients had a median age of 25 years (age range, 18 to 55 years). The most common presenting complaint was chest pain, occurring in 17 patients (85\%). Dyspnea was the next most common complaint (13 patients-65\%). Other presenting complaints included neck pain, swelling, and hoarse voice. Associated problems were mild asthma in seven patients, history of smoking in six patients, illicit drug use in two patients, and interstitial lung disease in one patient. The latter patient was an old female, who normally received therapy with oral corticosteroids for her lung disease and had experienced no specific precipitating event prior to the onset of her symptoms. The patients reported precipitating events in only half of the presentations which included coughing, sport activity and mild trauma.

The initial diagnosis was almost correctly made in four patients $(20 \%)$ and in the remaining patients, the diagnosis was thought to be esophageal rupture, cardiac events and pneumothorax. The most common physical finding on presentation was subcutaneous emphysema. Hamman sign, which is a crunching sound in time with the heartbeat, was noted in only two patients (10\%). All of the patients had undergone radiologic investigation including Chest $\mathrm{X}$ ray and CT Scan. Cardiologist consultation was also required for most patients. All patients were admitted to the hospital and observed for a period of time, while one patient was admitted to the ICU. All patients survived and were discharged from the hospital after a median time of 2 days (range, up to 4 days). Most patients were seen early after their hospital discharge with normal findings on a chest radiograph. There is no record of any of these patients having a recurrence of their spontaneous pneumomediastinum.

\section{Discussion}

Pneumomediastinum is the presence of extraluminal gas within the mediastinum. Gas may originate from the lungs, trachea, central bronchi, esophagus, and track from the mediastinum to the neck or abdomen. Rarely, dissection can escape the soft tissues and invade the visceral or parietal pleura of the lungs, leading to pneumothorax. Case reports have also described spontaneous dissection into the peritoneum and pericardium, leading to pneumoperitoneum and pneumopericardium, respectively. Spontaneous pneumomediastinum (SPM) has been described predominantly in young adult men, and its incidence appears to be increasing in more recent reports, but this may reflect greater physician awareness. Macklin and Macklin initially described the pathophysiology of this condition in 1944 based on the results of animal experiments. The underlying factor was terminal alveolar rupture into the lung interstitium secondary to increased alveolar pressure or overdistension and the dissection of that gas into the hilum and subsequently the mediastinum along a pressure gradient.
From there, the gas follows the fascial planes, often into the tissues of the neck. Spontaneous pneumomediastinum had been associated with activities that result in the Valsalva maneuver such as childbirth, forceful straining during exercise, straining at stool, coughing, sneezing, retching, or vomiting, pulmonary function testing and inflation of party balloons. Asthma exacerbation, COPD, and bronchitis are the most commonly reported triggers associated with pneumomediastinum, accounting for up to $40 \%$ to $50 \%$ of reported cases [5]. A history of asthma has been reported as a factor in the development of SPM in up to $50 \%$ of cases, and in this series we had $30 \%$ of patients with a recent or remote history of asthma. Other conditions associated with SPM include Acute laryngitis, Interstitial lung disease, Peptic ulcer disease, Anorexia nervosa and Panic attack. Physical exam in these patients is often unremarkable with relatively normal vital signs. Hamman's sign (crepitus heard with the heartbeat upon auscultation of the chest) is rarely appreciated on physical exam.

Two authors have reported EKG abnormalities associated with pneumomediastinum. Mondello et al. [6] note that lowamplitude QRS may be observed, while Macia et al. [7] report STsegment elevation within part of their cohort. Regarding radiologic studies up to $30 \%$ of cases may be missed on chest radiograph alone [8-10]. CT of the chest is considered to be the gold standard imaging modality. A recent series from Bakhos et al. suggests that esophagography or other invasive endoscopic testing should not be used routinely [5]. They outline risk factors which should prompt further investigation in select cases: age $>40$ years, history of severe vomiting or retching, abdominal pain on exam, elevated white blood cell count, or concerning finds on chest CT such as pleural effusion, pneumopericardium, pneumoperitoneum, or significant atelectasis. Once the clinician has excluded serious causes and has arrived at the diagnosis of primary pneumomediastinum, management is directed toward supportive care and symptom relief, monitoring for potential complications, and treatment of underlying etiology. In a recent series, Lee and colleagues recommend that conservative management with hospitalization less than 24 hours is both safe and feasible [11]

\section{Conclusion}

The most important role of thoracic surgeon is to exclude the absence of more serious abnormalities, especially those who have a history of vomiting or retching. Boerhaave syndrome must be considered in any person who has a history of vomiting followed by chest pain with dyspnea. All of these patients should be observed for a period of time in a hospital setting to exclude the development of complications.

\section{References}

1. Macklin CC (1939) Transport of air along sheathes of pulmonic blood vessels from alveoli to mediastinum. Arch Int Med 64: 913-926.

2. Macklin MT, Macklin CC (1944) Malignant interstitial emphysema of the lungs and mediastinum as an important occult complication in many 
respiratory diseases and other conditions: interpretation of the clinical literature in the light of laboratory experiment. Medicine 23(4): 281358.

3. Newcomb AE, Clarke CP (2005) Spontaneous pneumomediastinum: a benign curiosity or a significant problem? Chest 128(5): 3298-3302.

4. Sahni S, Verma S, Grullon J, Esquire A, Patel P, et al. (2013) Spontaneous pneumomediastinum: time for consensus. N Am J Med Sci 5(8): 460-464.

5. Bakhos CT, Pupovac SS, Ata A, Fantauzzi JP, Fabian T (2014) Spontaneous pneumomediastinum: an extensive workup is not required. J Am Coll Surg 219(4): 713-717.

6. Mondello B, Pavia R, Ruggeri P, Barone M, Barresi P, et al. (2007) Spontaneous pneumomediastinum: experience in 18 adult patients. Lung 185(1): 9-14.

7. Macia I, Moya J, Ramos R, Morera R, Escobar I, et al. (2007) Spontaneous pneumomediastinum: 41 cases. Eur J Cardio Thorac Surg 31(6): 1110 1114.

ISSN: 2574-1241

DOI: 10.26717/BJSTR.2019.20.003419

Mohammad Vaziri. Biomed J Sci \& Tech Res

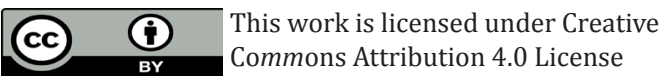

Submission Link: https://biomedres.us/submit-manuscript.php
8. Caceres M, Ali SZ, Braud R, Weiman D, Garrett HE (2008) Spontaneous pneumomediastinum: a comparative study and review of the literature. Ann Thorac Surg 86(3): 962-926.

9. Campillo Soto A, Coll Salinas A, Soria Aledo V, Blanco Barrio A, Flores Pastor B, et al. (2005) Spontaneous pneumomediastinum: descriptive study of our experience with 36 cases. Arch Bronconeumol 41(9): 528531.

10. Kaneki T, Kubo K, Kawashima A, Koizumi T, Sekiguchi M, et al. (2000) Spontaneous pneumomediastinum in 33 patients: yield of chest computed tomography for the diagnosis of the mild type. Respir Internat Rev Thorac Dis 67(4): 408-411.

11. Lee SC, Lee DH, Kim GJ (2014) Is primary spontaneous pneumomediastinum a truly benign entity? Emerg Med Australas 26(6): 573-578.

$\begin{array}{ll}\text { BIOMEDICAL } & \text { Assets of Publishing with us } \\ \text { RESEARCHES } & \text { - Global archiving of articles } \\ & \text { - Immediate, unrestricted online access } \\ \end{array}$

